Proceedings

\title{
Development of Wireless Monitoring System for Pulse Rate: A New Approach +
}

\author{
Mohammad Monirujjaman Khan *, Tahia Tazin and Tabia Hossain \\ Department of Electrical and Computer Engineering, North South University, Bashundhara, Dhaka-1229, \\ Bangladesh \\ * Correspondence: monirujjaman.khan@northsouth.edu; Tel.: +8-801-779-006-296 \\ † Presented at 1st International Electronic Conference on Applied Sciences, 10-30 November 2020; Available \\ online: https://asec2020.sciforum.net/.
}

Published: 10 November 2020

\begin{abstract}
Various types of heart diseases including cardiac arrhythmia, myocardial infarction, and coronary artery disease are one of the main reasons behind the causes of death around the world. It can be mitigated if we know the pulse rate and monitor it properly. But constant monitoring can be expensive for the private sector and so we are proposing to solve the problem by the implementation of a wireless network based on Bluetooth. The pulse rate data is sent from Arduino Uno via Bluetooth to Smartphone and it can be analyzed by the user and sent it to an expert doctor with a little cost and more efficiently. This project identifies with a heartbeat rate estimation gadget including a heartbeat rate sensor unit that distinguishes a client's heartbeat rate, a sign preparing unit that gets and measures the sign produced from the sensor, and a remote sign sending unit that takes the sign from the handling unit and the communication the sign out to the arranged gadget. The sensor unit distinguishes the recurrence of the progress of blood thickness to get the pulse, carefully and productivity of location of pulse, participating in the method of remote transmission, and accordingly our motivation of advancing precision of identification and improving comfort of utilizing is accomplished. The model incorporates Arduino Uno, Pulse Rate Sensor, Bluetooth Board, Breadboard, USB link, and so forth we communicate the information utilizing Bluetooth to Smartphone utilizing Pulse Rate Monitor circuit furnished with Arduino Uno.
\end{abstract}

Keywords: Wireless Monitoring; Pulse Rate; Arduino Uno; Smartphone; Sensor Units; Heart Diseases; Bluetooth; Breadboard; Medical

\section{Introduction}

These days, individuals influenced by experiencing cardiovascular sicknesses are expanding step by step. So here the desire emerges to plan a viable framework that would give us exact and speedy heartbeat rate readings [1-3]. The readings are communicated to the associated cell phone through Bluetooth for example through remote media. By giving admittance to a cell phone and heartbeat rate circuit we eliminate the obstructions of specialists and patients, offer clinical types of assistance, and outfit the patient with such innovation that they can get their heartbeat rate information with a cell phone and send the information to an expert specialist or paramedic who can get them out with different tips what to do during arrhythmias or other cardiovascular issue or season of crisis mediation of paramedics such circumstance as in myocardial localized necrosis [4]. In-home wellbeing, the utilization of innovation that permits patients to screen their fundamental signs from their home and impart results to a medical clinic proficient remotely could build the capacity to address an issue before a patient requires intense consideration.Heartbeat is the rate at which somebody's pulses. Heartbeat rate is normally called your pulse, which is the occasion pulses every moment (bpm). In any case, the beat and quality of the heartbeat can likewise be noted, just as Proceedings 2020, 2020 www.mdpi.com/journal/proceedings 
whether the vein feels hard or delicate. Changes in somebody's pulse or mood, a frail heartbeat, or a hard vein might be brought about by coronary illness or another issue. As the heart siphons blood through your body, you can feel a beating in a portion of the veins near the skin's surface, for example, in your wrist, neck, or upper arm. The tallying beat rate is a straightforward method to discover how quickly your heart is pulsating [4].

\section{System Design}

This research identifies with a heartbeat rate estimation gadget containing a heartbeat rate sensor unit that distinguishes a client's heartbeat rate, a sign preparing unit that gets and measures the sign created from the sensor, and a remote sign sending unit that takes the sign from the handling unit and the communication the sign out to the designed gadget. The sensor unit unequivocally recognizes the recurrence of progress of blood thickness to get the pulse, carefully, and productivity of discovery of pulse, participating in the method of remote transmission, and subsequently, our motivation of advancing exactness of location and improving the comfort of utilizing is accomplished. The model incorporates Arduino Uno, Pulse Rate Sensor, Bluetooth Board, Breadboard, USB link, and so forth we communicate our information through Bluetooth to cell phones utilizing Pulse Rate Monitor circuit furnished with Arduino Uno.

\section{Hardware and Software}

This system has been created by using Arduino Uno, Pulse sensor, Bluetooth HC-05 class, breadboard, and jumper wires.

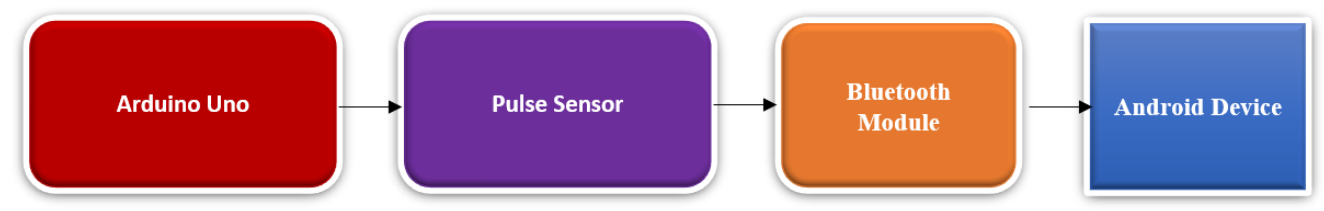

Figure 1. Flow-diagram of the system.

The heartbeat sensor depends on the rule of photoplethysmography [5-9]. It quantifies the adjustment in volume of blood through any organ of the body which causes an adjustment in the light force through that organ (avascular area). On account of uses where the heartbeat rate is to be observed, the circumstance of the beats is more significant. The progression of blood volume is chosen by the pace of heartbeats and since light is consumed by the blood, the sign heartbeats are equal to the heartbeat beats. There are two sorts of photoplethysmography.

- Transmission: Light discharged from the light-producing gadget is sent through any vascular area of the body like the ear cartilage and got by the locator.

- Reflection: Light produced from the light-radiating gadget is reflected by the locales.

We have utilized two heartbeat rate sensors; one is a modest Chinese variation and another unique heartbeat rate sensor from SparkFun (USA). The two sensors worked productively. Information we got from the US variant is steadier and more dependable.

The fundamental heartbeat sensor comprises of a light-emanating diode and an indicator like a light recognizing resistor or a photodiode. The heartbeat beats make a variety in the progression of blood in various areas of the body. At the point when a tissue is lit up with the light source, for example, light discharged by the drove, it either mirrors (a finger tissue) or communicates the light (ear cartilage). A portion of the light is consumed by the blood and the sent or the mirrored light is gotten by the light indicator. The measure of light retained relies upon the blood volume in that tissue. 


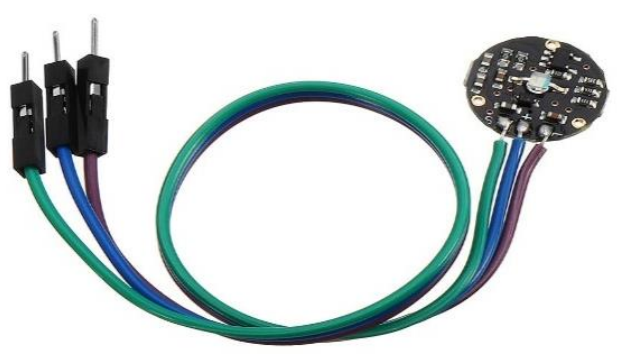

Figure 2. Pulse Sensor [10].

The indicator yield is in the type of electrical sign and is relative to the heartbeat rate. This sign is a DC signal identifying with the tissues and the blood volume and the AC part simultaneous with the heartbeat and brought about by pulsatile changes in blood vessel blood volume is superimposed on the DC signal. Subsequently, the significant necessity is to detach that AC part for what it's worth of prime significance. To accomplish the undertaking of getting the AC signal, the yield from the identifier is first sifted utilizing a 2 phase HP-LP circuit and is then changed over to advanced heartbeats utilizing a comparator circuit or utilizing basic ADC. The advanced heartbeats are given to a microcontroller for ascertaining the heartbeat rate, given by the recipe BPM (Beats every moment $)=60^{*} \mathrm{f}$; Where $\mathrm{f}$ is the beat recurrence.

Arduino/Genuino [11] Uno is a microcontroller board dependent on the ATmega328P. It has 14 advanced info/yield pins (of which 6 can be utilized as PWM yields), 6 simple information sources, a $16 \mathrm{MHz}$ quartz precious stone, a USB association, a force jack, an ICSP header, and a reset button. It contains an architect expected to help the microcontroller; just by interfacing it to a PC with a USB link or force it with an AC-to-DC connector or battery to begin. $b$ The Uno contrasts from all former sheets in that it doesn't utilize the FTDI USB-to-chronic driver chip. All things considered, it includes an ATmega16U2 customized as a USB-to-chronic converter. This helper microcontroller has its USB bootloader, which permits progressed clients to reconstruct it.

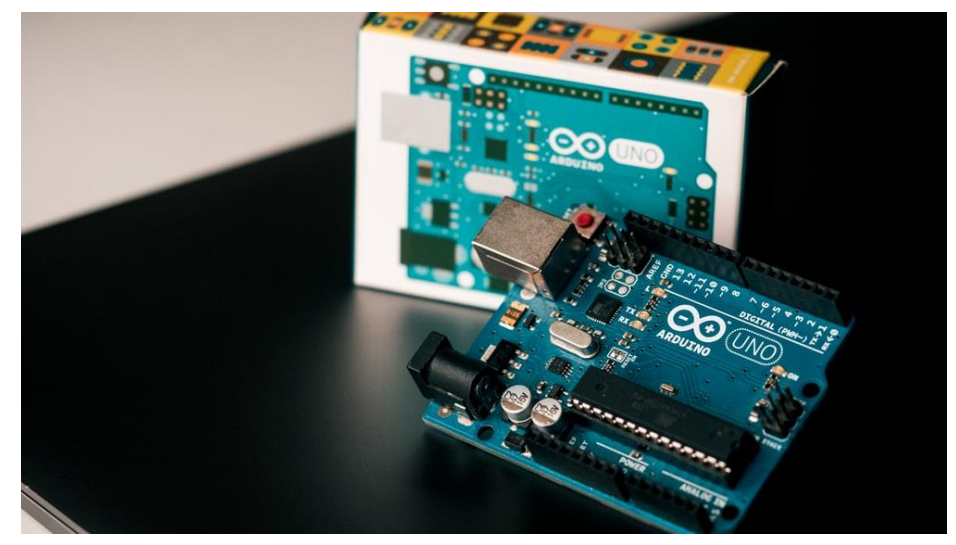

Figure 3. Arduino Uno [12].

HC-05 module is a simple to utilize Bluetooth SPP (Serial Port Protocol) module, intended for straightforward remote sequential association arrangement. The HC-05 Bluetooth Module can be utilized in a Master or Slave arrangement, making it an incredible answer for remote correspondence. This sequential port Bluetooth module is a completely qualified Bluetooth V2.0+EDR (Enhanced Data Rate) 3Mbps Modulation with a complete $2.4 \mathrm{GHz}$ radio handset and baseband. It utilizes CSR Bluecore 04-External single-chip Bluetooth framework with CMOS innovation and with AFH (Adaptive Frequency Hopping Feature). 


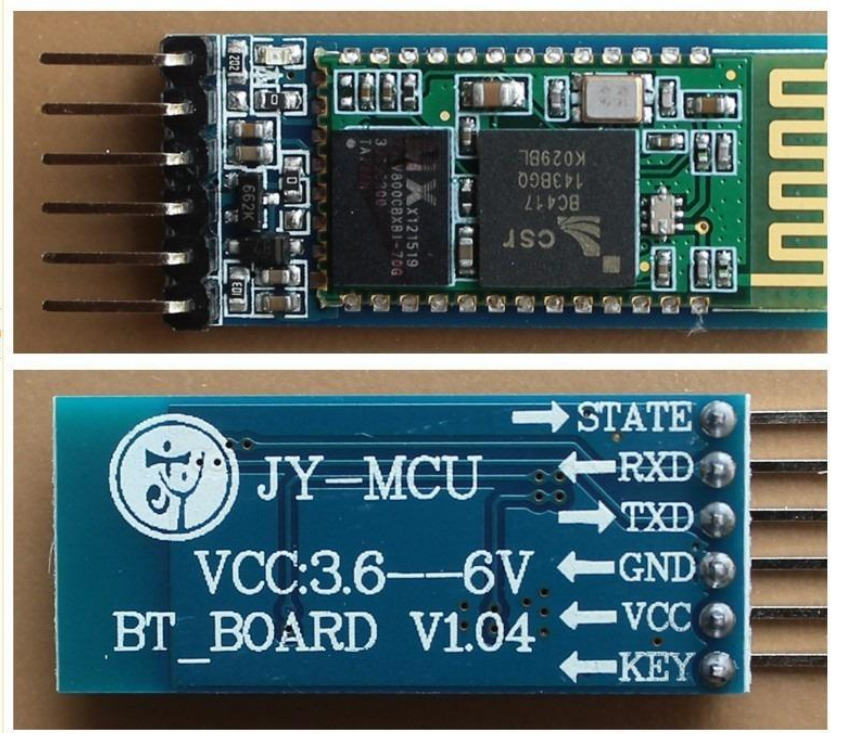

Figure 4. Bluetooth module [13].

\section{Result Analysis}

We utilized an open-source programming language/advancement instrument for composing programs on different PCs. Valuable when we need the PCs to converse with Arduino. The android application is local and we have utilized java and XML for this. We have utilized Bluetooth to get the information from the gadget and afterward we have utilized this information to draw the chart. It would take the perusing from the Arduino and show it on NSU_PRM that we explicitly produced for that reason. Screen capture of the application given underneath.

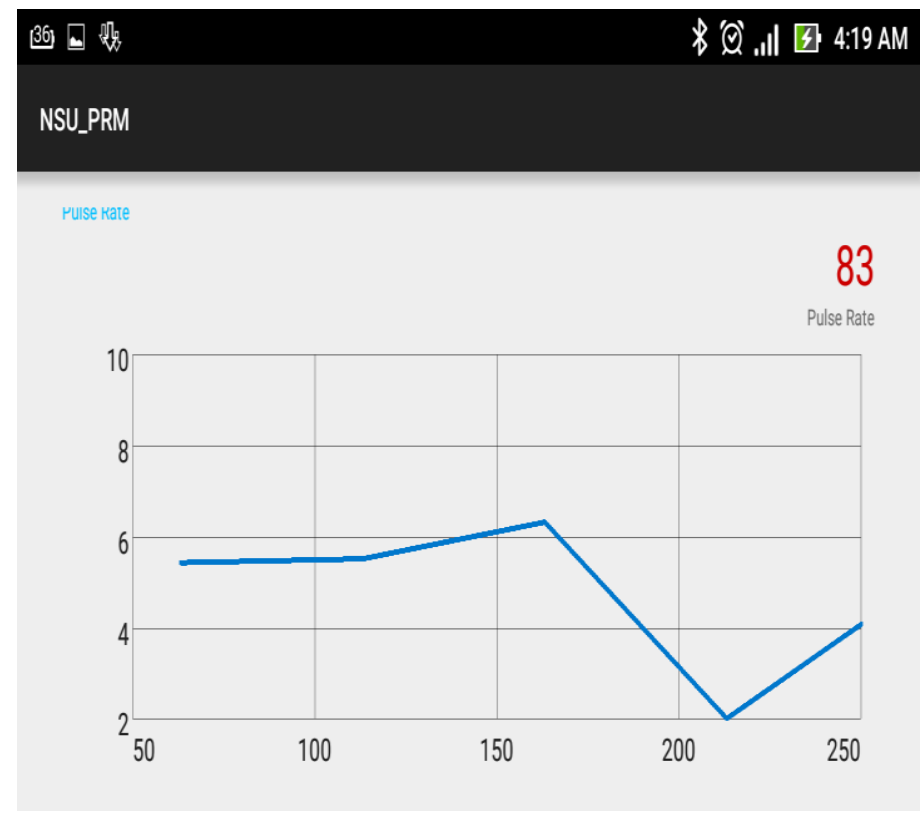

Figure 5. Android application interface.

This project identifies with a heartbeat rate estimation gadget involving a heartbeat rate sensor unit that distinguishes a client's heartbeat rate, a sign preparing unit that gets and measures the sign created from the sensor, and a remote sign communicating unit that takes the sign from the handling unit and the send the sign out to the arranged gadget. The sensor unit accurately distinguishes the recurrence of progress of blood thickness to get the pulse, fastidiously and productivity of discovery of pulse, co-working the procedure of remote transmission, and in this way, our motivation of advancing precision of location and improving accommodation of utilizing is accomplished. The 
model envelopes of Arduino Uno, Pulse Rate Sensor, Bluetooth Board, Breadboard, USB link, and so forth We send our information through Bluetooth to Smartphone utilizing Pulse Rate Monitor circuit outfitted with Arduino Uno.

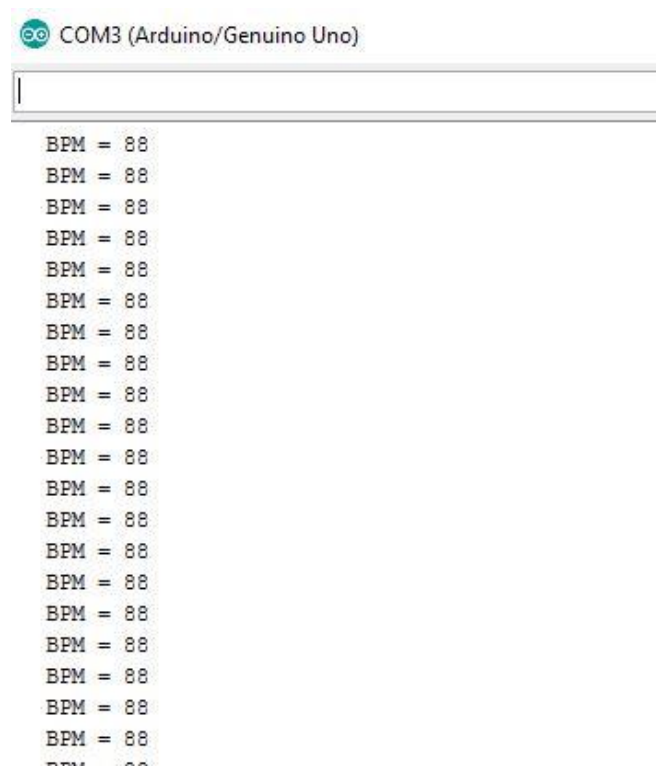

Figure 6. Pulse rate result in Arduino IDE.

We have estimated the beat pace of numerous volunteers both by our gadget circuit and physically. The two outcomes nearly agreed precisely. Heartbeat rate got a worth normal of around 67 to 80 BPM. What's more, we got appropriate an incentive on Arduino Uno and Android Apps. By dissecting the manual estimations of the heartbeat rate, we got a minimal blunder of $+/-2 \%$ as it were. So it agreed with our worth precisely.

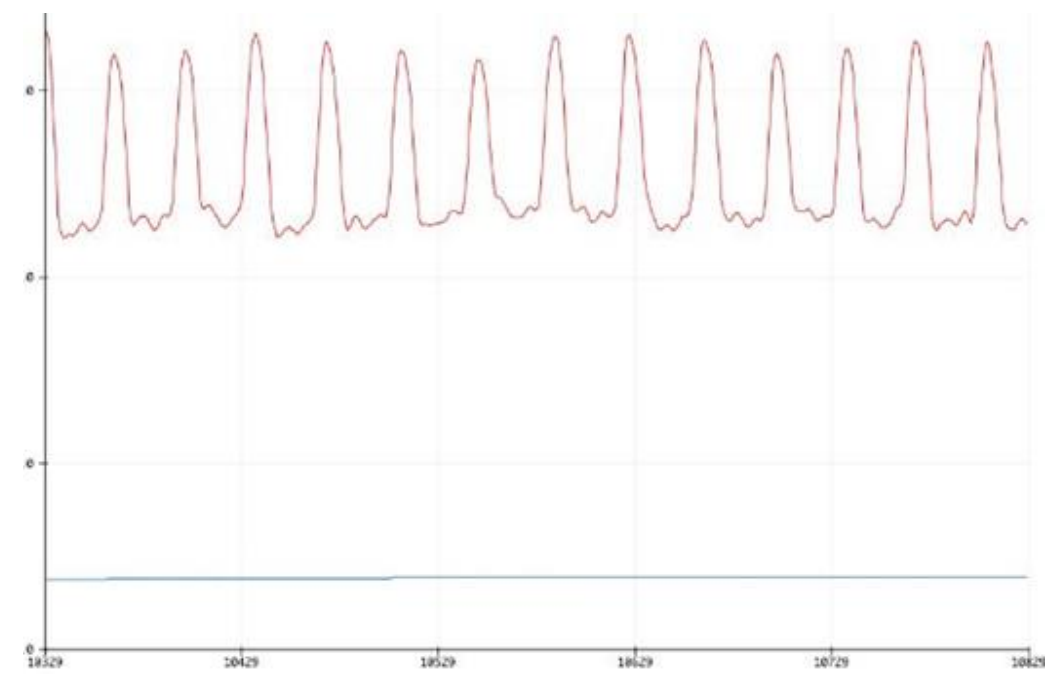

Figure 7. Arduino IDE plotter.

\section{Conclusion}

It has been effectively demonstrated through the model that works impeccably. These days, individuals influenced by experiencing heart infections are expanding step by step. So here an inclination emerges to plan a viable framework that would give us precise and brisk heartbeat rate readings The readings are sent to the associated cell phone through Bluetooth for example through remote media. By giving admittance to a cell phone and heartbeat rate circuit we eliminate the hindrances of specialists and patients, offer clinical types of assistance, and furnish the patient with 
such innovation that they can get their heartbeat rate information with a cell phone and send the information to a pro specialist or paramedic who can get them out with different tips what to do during arrhythmias or other cardiovascular issue or season of crisis mediation of paramedics such circumstance as in myocardial localized necrosis. In-home wellbeing, the utilization of innovation that permits patients to screen their imperative signs from their home and impart results to a medical clinic proficient remotely could expand the capacity to address an issue before a patient requires intense consideration. In this paper, we have executed a remote heartbeat rate screen with Arduino Uno, Bluetooth Module, and Pulse Sensor and so forth the venture has effectively appeared and it shows a diagram of heartbeat rate remotely in the Android application through Bluetooth gadget. We have estimated the beat pace of numerous volunteers both by our gadget circuit and physically. The two outcomes nearly agreed precisely. Heartbeat rate got a worth normal of around 67 to 80 BPM.

Funding: This research received no external funding.

\section{References}

1. Mensink, G.B.M.; Hoffmeister, H. The relationship between resting heart rate and all-cause, cardiovascular, and cancer mortality. Eur. Heart J. 1997, 18, 1404-1410.

2. Kannel, H.W.B.; Kannel, C.; Paffenbarger, R.S.; Cupples, L.A. Heart rate and cardiovascular mortality: the Framingham Study. Am. Heart J. 1987, 113, 1489-1494.

3. Thaulow, E.; Eriksson, J.E. How important is heart rate? J. Hypertens. 1991, 9 (Suppl. 7), S17-S23.

4. Landicho, L.C.L.; Magbalon, I.R.; Reyes, C.P.S. "A wireless electrocardiography in superintending cardiac rate extremes with global positioning capability to remotely localize specimen." In Proceedings of the 2015 IEEE International Conference on ControlSystem, Computing and Engineering (ICCSCE), George Town, Penang, Malaysia, 27-29 November, 2015; pp. 141-145.

5. Reyes, I.; Nazeran, H.; Franco, M.; Haltiwanger, E. “Wireless photoplethysmographic device for heart rate variability signal acquisition and analysis." In Proceedings of the 2012 Annual International Conferenceof the IEEE Engineering in Medicine and Biology Society, SanDiego, CA, USA, 28 August-1 September 2012; pp. 2092-2095.

6. Rotariu, C.; Manta, V. "Wireless system for remote monitoring of oxygen saturation and heart rate." In Proceedings of the 2012 FederatedConference on Computer Science and Information Systems (FedCSIS), Wroclaw, Poland, 9-12 September 2012; pp. 193-196.

7. Cristea, C.; Pasarica, A.; Andruseac, G.; Dionisie, B.; Rotariu, C. “A wireless ECG acquisition device for remote monitoring of heart rate and arrhythmia detection." In Proceedings of the 2015 E-Health and Bioengineering Conference (EHB), Iasi, Romania. 19-21 November 2015; pp. 1-4.

8. Jubadi, W.M.; AisyahMohdSahak, S.F. "Heartbeat monitoring alert via SMS." In Proceedings of the 2009 IEEE Symposium on IndustrialElectronics \& Applications, Kuala Lumpur, Malaysia, 4-6 October 2009; pp. $1-5$.

9. Leijdekkers, V.G.P. A Health Monitoring System Using Smart Phones and Wearable Sensors. Int. J. ARM 2007, 8, 29-36.

10. Pulse Sensor. Available online: https://www.banggood.com/Pulsesensor-Pulse-Heartbeat-Rate-Sensor-Module-Pulse-Sensor-p-1327349.h tml?akmClientCountry=BD\&\&cur_warehouse $=\mathrm{CN}$ (accessed on 5 May 2020).

11. “Arduino-ArduinoBoardUno", Arduino.cc, 2017.

12. Arduino Uno. Available Online: https://unsplash.com/s/photos/arduino-uno (accessed on 5 May 2020)

13. Bluetooth Module. Available Online: https://unsplash.com/s/photos/arduino-uno (accessed on 5 May 2020).

Publisher's Note: MDPI stays neutral with regard to jurisdictional claims in published maps and institutional affiliations.

(C) 2020 by the authors. Submitted for possible open access publication under the terms and conditions of the Creative Commons Attribution (CC BY) license (http://creativecommons.org/licenses/by/4.0/). 